\title{
Evaluar los enemigos naturales de Leucaena Psyllid y Onion Thrips en los procesos de cosecha en diferentes genotipos de Leucaena*
}

\author{
Survey the natural enemies of Leucaena Psyllid and Onion Thrips \\ on the harvest processes in different Leucaena genotypes
}

\begin{abstract}
Ahmed M. M. Ahmed ${ }^{1.2 \S}$, Francisco J. Solorio Sánchez ${ }^{1}$, Luis Ramírez y Avilés ${ }^{1}$ y Al-Zyoud, F. A. ${ }^{3}$
${ }^{1}$ Facultad de Ciencias de Medicina Veterinaria y Zootecnia-Universidad de Yucatán, Mérida, Yucatán, México. ${ }^{2}$ Department of Plant Protection, Faculty of Agriculture, Assiut University, Assiut (71526), Egypt. ${ }^{3}$ Department of Plant Protection and Integrated Pest Management, Faculty of Agriculture, Mu’tah University, Karak P. O. BOX 61710, Jordan. ${ }^{\S}$ Corresponding author: saleh_moda@yahoo.com.
\end{abstract}

\section{Resumen}

El estudio se llevó a cabo en 2013 y 2014, en Xmatkuil, Mérida, Yucatán, México a la encuesta enemigos naturales y sus efectos sobre la dinámica poblacional de Leucaena leucocephala (Lam.) de plagas Wit: Leucaena Psílidos, Heteropsylla cubana Crawford (1914) (Homoptera: Psyllidae) y cebolla trips, Thrips tabaci Lindeman, 1889 (Thysanoptera: Thripidae) en cuatro genotipos de Leucaena: Cunningham, K636, Nativa y KX2. Se recogieron las hojas juveniles, examinaron y cuenta del enemigo natural, se correlacionaron con cada uno de los números de plagas. Nueve depredadores relacionados con 5 órdenes, y uno parasitoide controlan las poblaciones de ambas plagas. En la primera temporada Nativa tuvo las mayores densidades de Psyllid, y Cunningham para Thrips. En la segunda temporada, Nativa para ambas plagas. Los números de menos registran en KX2 para ambas plagas. El coeficiente de correlación (r) fue más fuerte entre los enemigos naturales y $H$. cubana que T. tabaci. En la primera temporada se produjeron tres picos del psílido, dos antes de la primera cosecha en mayo y junio y una después en diciembre. De lo contrario, cuatro picos de trips se registraron en Cunningham, Nativa y K636 durante mayo a agosto y sólo dos ocurrieron en KX2 en mayo yjunio. En la segunda temporada de la mayor población de plagas

\section{Abstract}

The study was conducted in 2013 and 2014, at Xmatkuil Merida, Yucatan, Mexico to survey natural enemies and their effects on the population dynamics of Leucaenaleucocephala (Lam.) de Wit pests: Leucaena Psyllids, Heteropsylla cubana, Crawford (1914)(Homoptera: Psyllidae) and Onion Thrips, Thrips tabaci, Lindeman (1889) (Thysanoptera: Thripidae) on four Leucaena genotypes: Cunningham, K636, Nativa and KX2. The juvenile leaves were collected, examined and the natural enemy's counts were correlated with each pest numbers. Nine predators related to 5 orders, and one parasitoid controlled the populations of both pests. In the first season Nativa had the largest densities of Psyllid, and Cunningham for Thrips. In the second season, Nativa for both pests. The least numbers recorded in KX2 for both pests. The coefficient of correlation (r) was stronger between natural enemies and H. cubana than T. tabaci. In the first season three peaks of Psyllid were occurred, two before the first harvest during May and June and one after at December. Otherwise, four peaks of Thrips were recorded on Cunningham, Nativa, and K636 during May to Aug. and only two occurred on KX2 at May and June. In the second season the largest population of pests was recorded in Nativa, and the lowest were in KX2 for both pests ( $\mathrm{r}$ ) was negative

\footnotetext{
* Recibido: septiembre de 2015

Aceptado: enero de 2016
} 
se registró en Nativa, y la más baja se encontraban en KX2 para ambas plagas (r) fue negativa entre trips y enemigos naturales. Psyllid mostró dos picos en febrero y abril, y dos para los trips en abril y junio.

Palabras clave: control biológico, dinámica de Leucaena plagas, Leucaena psílido, trips de la cebolla.

\section{Introducción}

Sin lugar a dudas, Leucaena leucocephala (Lam.) de Wit, (leguminosae: Mimosoideae) condujo a una revolución en el suministro de alimentos de origen animal. En el pasado, Leucaena era conocida por la ausencia de enfermedades, plagas de insectos, y esa fue la razón detrás de su difusión en el mundo tropical. Pero, recientemente, Leucaena fue más vulnerable a las plagas de insectos plaga. Estas plagas se dividen en plaga mayor y menor de acuerdo con el rango de hospederos y la interacción entre las plagas y Leucaena ya sea monofago u oligofago.

Elmonofago H.cubana es una plagaimportante quesealimenta exclusivamente de L. leucocephala, y en menor medida, de árboles de mimosa en varias regiones del mundo. La plaga es nativa de América Central y Sur y se extendió a África, Asia y provoca grandes daños principalmente a Leucaena Heydon y Affonso(1991); USAID(1991); FAO(2007); Raoetal.(2000). Es un ejemplo típico de riesgo de plagas para sus devastadores brotes en plantaciones exóticas de L. leucocephala a través de los trópicos (Nair, 2007). Además, alcanzó notoriedad internacional en la década de 1980 cuando se detectó en Florida y Hawaii con una velocidad de propagación extremadamente rápida. Por lo tanto, es razonable suponer que tarde o temprano todas las áreas donde se cultiva Leucaena se verán afectadas (Bray 1994; Geiger et al., 1995; Olckers, 2011).

El oligofago T. tabaci es una plaga menor por su capacidad de completar su ciclo de vida en diferentes plantas hospederas a Leucaena, lo que hace que sea difícil de controlar. $T$. tabaci ha sido registrada en más de 300 especies de plantas hospederas de diferentes grupos (Sakimura 1932; Ghabn, 1948). En este trabajo se registró como una plaga menor en Leucaena por primera vez.

En el ámbito del manejo de plagas, dejar de usar plaguicidas en árboles forrajeros y usar agentes de control biológico sigue siendo la primer demanda en todo el mundo, ya between Thrips and natural enemies. Psyllid showed two peaks in February and April, and two for Thrips in April and June.

Keywords: biological control, Leucaena Psyllid, Leucaena pest's dynamics, onion thrips.

\section{Introduction}

Undoubtedly, Leucaena leucocephala (Lam.) de Wit, (leguminosae: Mimosoideae) led to a revolution in animal food supplies. In the past, Leucaena was known by the absence of disease, insect pest's infestation, and that was the reason behind its spread in the tropical world. But, recently Leucaena was more vulnerable to insect pests infestation. These pests are divided into major and minor pest according to the host range and the interaction between pest and Leucaena whether monophagous or oligophagous pest.

The monophagous, H. cubana is a major pest which exclusively feeds on $L$. leucocephala and to a lesser extent on closely related mimosoid leguminous trees at several world regions. The pest is native to Central and South America and spread to Africa, Asia and mainly exists with extensive damage to Leucaena Heydon and Affonso (1991); USAID (1991); FAO (2007); Rao et al. (2000). It is a typical example of pest risk for its devastating outbreaks in exotic L. leucocephala plantations across the tropics (Nair, 2007). Besides; it achieved international notoriety in the early 1980 s when detected in Florida and Hawaii with an extremely rapid rate of spread. Therefore, it is reasonable to assume that sooner or later all areas where Leucaena is grown will be affected (Bray 1994; Geiger et al., 1995; Olckers 2011).

The oligophagous, T. tabaci is a minor pest for its ability in completing its life cycle on different alternative host plants than Leucaena, the thing that makes it difficult to be controlled. Previously, T. tabaci has been recorded from more than 300 species of host plant scattered through diverse plant groups (Sakimura 1932; Ghabn 1948). In this work it is recorded as a minor pest on Leucaena for the first time.

In the field of pest management, quitting pesticides with such animal fodder tree and using biological control agent still the first demand around the world, because it automatically regulates pest population such as the predators of Coleoptera: Coccinellidae: Curinus coeruleus 
que regula automáticamente poblaciones plagas como los depredadores de Coleoptera: Coccinellidae: Curinus coeruleus (Mulsant, 1850) Valenciaga et al. (1999); Singh (2004), Olla v-nigrum (Mulsant, 1866) y los parasitoides de Hymenoptera: Psyllaephagus yaseeni Noyes, 1990 y Tamarixia leucaena Bouček, 1988, los cuales han tenido éxito parcial (Napompeth 1994; Shivankar et al., 2010).

Estudio de la dinámica poblacional de plagas de Leucaena, $H$. cubana y T. tabaci bajo el efecto de sus enemigos naturales se considera como un factor primordial para realizar recomendaciones de manejo de plagas. Desde este punto de vista, la evaluación de los enemigos naturales de Leucaena es unimportante paso para estudiar sus efectos sobre la dinámica poblacional de las plagas más peligrosas para Leucaena.

\section{Materiales y métodos}

El presente experimento se llevó a cabo a lo largo de dos ciclos (2013 y 2014) en un campoubicado en la comunidad Xmatkuil en Mérida, Yucatán, México. El campo experimental (Figura 1) tiene cuatro parcelas (repeticiones), el cual se mantuvolibre de malezas a través de deshierbe manual sin usar herbicidas. El tamaño de las parcelas fue de $9 \mathrm{~m} \times 6 \mathrm{~m}$, cada genotipo se plantó en una hilera (cuatro hileras por parcela), el espacio entre hileras es de $2 \mathrm{~m}$, y $1 \mathrm{~m}$ entre árboles. Cada genotipo estuvo conformado de 10 árboles por hilera. En el primer ciclo (2012-2013) al comienzo del experimento, los árboles tenían (2 años y 3 meses de edad) y no fueron cosechados hasta el final de octubre de 2013. Subsecuentemente, las cosechas se realizaron cada dos meses para evaluar el efecto de la composición química de nuevas hojas durantelos meses de la segunda temporada (2013-2014). Se dejaron a crecer por un mes los árboles, para tomar muestras en el segundo mes. La cosecha se realizó cuando la planta alcanzó $1 \mathrm{~m}$ de altura, de acuerdo con los procesos de agricultura tradicional de Leucaena en Mérida, Yucatán, México. Las cosechas se realizaron al final de los siguientes meses: primera cosecha en octubre de 2013; segunda cosecha diciembre 2013; tercera cosecha febrero 2014; cuarta cosecha abril 2014; quinta cosecha junio 2014, y sexta cosecha agosto 2014. Para evaluar los enemigos naturales de las plagas más abundantes de Leucaena: H. cubana y T. tabaci, y estudiar sus efectos sobre la fluctuación poblacional en hojas nuevas y viejas de Leucaena en cuatro genotipos de Leucaena: dos cultivares de Leucaena, Cunningham y K636 (L. leucocephaIa), y dos híbridos Nativa y KX2 (L. leucocephala x L. pallida).
(Mulsant, 1850) Valenciaga et al. (1999); Singh (2004), Olla v-nigrum (Mulsant, 1866) and the parasitoids of Hymenoptera: Psyllaephagus yaseeni Noyes, 1990 and Tamarixia leucaenae Boucek, 1988 which have met with partial success (Napompeth 1994; Shivankar et al. 2010).

Study of Leucaena pest's population dynamics, H. cubana and T. tabaci under the effect of their natural enemies is considered as a corner stone to set management recommendations for pest control. From this point, survey of natural enemies which habitLeucaena is an important step to study their effects on the population dynamics of the most dangerous Leucaena pests.

\section{Materials and methods}

The present experiment was conducted throughout two seasons (2013 and 2014) at farm located in Xmatkuil community at Merida, Yucatan, Mexico. The experimental farm (Figure 1) contains four plots (replications), which maintained free weeds by hand weeding without using weedicides. The plots size is $9 \mathrm{mX} 6 \mathrm{~m}$, each genotype planted in row (four rows per plot), the spacing between rows is $2 \mathrm{~m}$, and $1 \mathrm{~m}$ within trees. Each genotype had 10 trees per row. In the first season (2012-2013) at the beginning of the experiment, the trees had ( 2 years and 3 months old) and weren't harvested till the end of October, 2013. Then, Successive harvests were done each two months in order to assess the effect of chemical compositions of new plant leaves during the months of the second season (2013-2014). The trees were allowed to grow in a month, and sampled in the second month. The harvest process took place at $1 \mathrm{~m}$ height from the soil according to the traditional agriculture processes of Leucaena at Merida, Yucatan, Mexico. Harvests were done at the end of the following months: $1^{\text {st }}$ harvest in October, $2013 ; 2^{\text {nd }}$ December $2013 ; 3^{\text {nd }}$ in February, 2014; $4^{\text {th }}$ in April, 2014; $5^{\text {th }}$ in June, 2014, and $6^{\text {th }}$ in August, 2014. To survey the natural enemies of the most abundant Leucaena pests: H. cubana and T. tabaci, and study their effects on the population fluctuation on new and old Leucaena leaves of four Leucaena genotypes: two cultivars of Leucaena, Cunningham and K636 (L. leucocephaIa), and two hybrids Nativa and KX2 (L. leucocephala x L. pallida). Two trees from each row were tagged to collect the samples from their terminal fresh leaves, as most of heavy infestations occurred in the terminal shoots; up to 3000 nymphs and adults were recorded in the terminal $(15 \mathrm{~cm})$ of shoot by Nair (2007), also the Psyllid populations boomed in the presence of young Leucaena leaves (Geiger and Andrew, 2000). The 
Se marcaron dos árboles de cada hilera para recolectar las muestras de hojas en yemas terminales, ya que la mayoría de las infestaciones severas ocurrieron en estos; Nair (2007), registro hasta 3000 ninfas y adultos en brote terminal $(15 \mathrm{~cm})$; de forma similar, las poblaciones de Psyllid aumentaron en presencia de hojas jóvenes de Leucaena (Geiger y Andrew 2000). Los árboles marcados fueron divididos en tres niveles superior, intermedio e inferior. Semanalmente serecolectaron dos hojas por nivel de cada árbol seleccionado (192 muestras por semana) libre de pesticidas.

Las hojas se cortaron cuidadosamente y se colocaron por separado en bolsas de plástico y almacenadas en refrigerador a 4-7 ${ }^{\circ} \mathrm{C}$, para su posterior evaluación el laboratorio de nutrición FMVZ de la Facultad de Ciencias de Medicina Veterinaria y Zootecnia (FMVZ) de la Universidad de Yucatán (UADY), Yucatán, México. Las cara superior e inferior de cada hoja fueron examinadas cuidadosamente bajo un microscopio [Nikon (SMZ 645), CW 10 x 22], y se registró el número de H. cubana y T. tabaci y sus enemigos naturales. Se ha dicho que el uso de agentes de control biológico es la mejor forma para controlar Leucaena Psyllid (Shivankar et al., 2010). Los enemigos naturales desconocidos se montaron en resina sintética y se enviaron a un especialista para su identificación y clasificación.

El coeficiente de correlación simple se calculó entre sumas de números medios mensuales de enemigos naturales y los números medios mensuales de cada plaga por separado en cada genotipo Leucaena (Cuadro 2).

\section{Resultados}

\section{Evaluación de enemigos naturales:}

Este estudio se centra en entender de manera global a los enemigos naturales de las plagas de Leucaena sobre los brotes nuevos de cuatro genotipos de Leucaena: Cunningham, Nativa, K636 y KX2. Los enemigos naturales evaluados con H. cubana y T. tabaci se muestran en la Cuadro 1. Los datos y observaciones de campo mostraron la presencia de 9 depredadores relacionados a 5 órdenes, y un parasitoide.

\section{Fluctuaciones poblacionales de H. cubana y $T$. tabaci}

Esta parte de la investigación actual se enfoca en la dinámica poblacional de las plagas insectos más peligrosos y abundantes, H. cubana y T. tabaci bajo el efecto de tagged trees were divided into three levels upper, middle and lower. Two leaves / level were collected weekly from each selected tree (192 samples per week) pesticides-free.

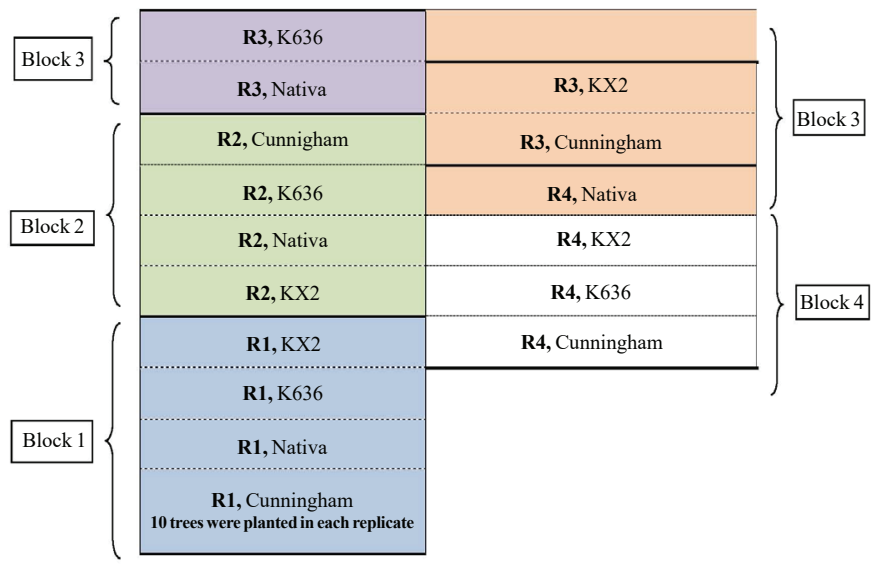

Figura 1. Distribución de los genotipos de Leucaena en los bloques experimental de la granja Xmatkuil, Mérida, Yucatán, México.

Figure 1. Distribution of Leucaena genotypes in the experimental blocks of Xmatkuil farm, Merida, Yucatan, Mexico.

The leaves were carefully cutand collected into separate plastic bags and transferred to the refrigerator and kept in fridge at $4-7^{\circ} \mathrm{C}$ forwell examination in the laboratory ofFMVZnutrition lab. at Faculty of Veterinary Medicine and Animal Science (FMVZ), University of Yucatan (UADY), Yucatán, Mexico. The upper and lower surfaces of each leaf were well examined by a binocular microscope [Nikon (SMZ645), C-W 10x22], and the numbers of $H$. cubana and T. tabaci and their natural enemies were recorded. It had been stated that using biological control agents is the best way to control Leucaena Psyllid (Shivankar et al., 2010). The unknown natural enemies were mounted on Resina Sintetica Media and sent to specialists for identification and classification.

The simple correlation coefficient was calculated between sums of monthly mean numbers of natural enemies and the monthly mean numbers of each pest separately in each Leucaena genotype (Table 2).

\section{Results}

\section{Survey of natural enemies:}

This study is focusing on a comprehensive understanding of the surveyed natural enemies of Leucaena pests on the new shoots of four Leucaena genotypes: Cunningham, Nativa, 
los enemigos naturales en diferentes genotipos de Leucaena (Cunningham, Nativa, K636 y KX2) se muestran en la Cuadro 2 y 3 e ilustrado gráficamente en Figura 2 y 3.
K636, and KX2. The surveyed natural enemies associated with H. cubana and T. tabaci is shown in Table 1. Data of survey study and frequent field observations indicated the presence of 9 predators related to 5 Orders, and one parasitoid.

Cuadro 1. Lista taxonómica parcial de $H$. cubana y T. tabaci enemigos naturales en cuatro genotipos de Leucaena; Cunningham y K636, Nativa y KX2 en el campo Xmatkuil durante los ciclos (2012-2013 y 2013-2014).

Table 1. A partial taxonomic list of $\boldsymbol{H}$. cubana and T. tabaci natural enemies on four Leucaena genotypes; Cunningham and K636, Nativa, and KX2 at Xmatkuil farm during (2012/13 -2013/14) seasons.

\begin{tabular}{ccccc}
\hline No. & Order & Family & Scientific name & N. E. type \\
\hline 1 & Neuroptera & Chrysopidae & Chrysoperla carnea (Stephens) & Predator \\
& & Coniopterygidae & Conwentzia barretti & Predator \\
2 & Hemiptera & Anthocoridae & Anthocoris nemoralis & Predator \\
3 & Coleoptera & Coccinelidae & Chilocorus stigma (Say) & Predator \\
& & & Olla v-nigrum Muslant & Predator \\
& & & Curinus coeruleus Mulsant & Predator \\
4 & Orthoptera & Mantidae & Archimantis latistyla (Serville) & Predators \\
& & & Mantis religiosa & Parasitoid \\
5 & Hymenoptera & Encyrtidae & Psyllaephagus yaseeni & Predator \\
\hline
\end{tabular}

N. E: Natural enemies.

Cuadro 2. Valores medios mensuales de las plagas de Leucaena $H$. cubana, T. tabaci y sus correlaciones con los enemigos naturales, en los cuatro genotipos de Leucaena en el campo Xmatkuil durante (2012-2013).

Table 2. Monthly mean numbers of leucaena pests $H$. cubana, T. tabaci and their correlations with natural enemies, on the four Leucaena genotypes at Xmatkuil farm during (2012-2013).

\begin{tabular}{cccccccccccccccc}
\hline $\begin{array}{c}\text { Sampling date } \\
\text { months }\end{array}$ & \multicolumn{4}{c}{ Cunningham } & \multicolumn{4}{c}{ Mean numbers of individuals } & \multicolumn{4}{c}{ K636 } & \multicolumn{4}{c}{ KX2 } & \multicolumn{3}{c}{ Grand mean } \\
& $\mathrm{H}$ & $\mathrm{T}$ & $\mathrm{N}$ & $\mathrm{H}$ & $\mathrm{T}$ & $\mathrm{N}$ & $\mathrm{H}$ & $\mathrm{T}$ & $\mathrm{N}$ & $\mathrm{H}$ & $\mathrm{T}$ & $\mathrm{N}$ & $\mathrm{H}$ & $\mathrm{T}$ \\
\hline Feb., 2013 & 0.0 & 0.0 & 0.0 & 0.0 & 0.0 & 0.0 & 0.0 & 0.0 & 0.0 & 0.0 & 0.0 & 0.0 & 0.0 & 0.0 \\
Mar. & 11.0 & 211.5 & 41.5 & 7.3 & 171.5 & 7.8 & 10.0 & 149.5 & 14.8 & 1.3 & 62.0 & 1.8 & 29.6 & 594.5 \\
Apr. & 159.0 & 544.8 & 171.8 & 137.8 & 372.5 & 158.3 & 87.0 & 258.0 & 114.0 & 95.8 & 272.5 & 79.5 & 479.6 & 1447.8 \\
May & 244.8 & 745.0 & 155.0 & 220.0 & 567.3 & 108.3 & 200.3 & 469.0 & 88.3 & 110.5 & 409.0 & 97.5 & 775.6 & 2190.3 \\
Jun. & 194.8 & 837.5 & 95.5 & 101.8 & 918.3 & 49.3 & 111.0 & 715.0 & 32.3 & 54.0 & 621.3 & 21.8 & 461.6 & 3092.1 \\
Jul. & 47.5 & 850.5 & 69.5 & 43.5 & 809.0 & 123.5 & 23.5 & 412.0 & 27.8 & 6.0 & 265.3 & 134.0 & 120.5 & 2336.8 \\
Aug. & 65.0 & 479.0 & 54.8 & 42.8 & 483.0 & 48.5 & 53.8 & 463.3 & 19.8 & 17.3 & 234.8 & 72.0 & 178.9 & 1660.1 \\
Sept. & 113.3 & 386.3 & 148.5 & 65.0 & 270.3 & 96.3 & 23.5 & 263.3 & 98.3 & 11.0 & 235.3 & 100.8 & 212.8 & 1155.2 \\
Oct. & 15.8 & 112.0 & 4.5 & 19.8 & 136.3 & 1.8 & 16.8 & 86.8 & 0.0 & 24.0 & 109.8 & 4.3 & 76.4 & 444.9 \\
Nov. & $*$ & $*$ & $*$ & $*$ & $*$ & $*$ & $*$ & $*$ & $*$ & $*$ & $*$ & $*$ & $*$ & $*$ \\
Dec., 2013 & 7422.3 & 0.0 & 0.0 & 8465.0 & 171.5 & 0.0 & 3866.0 & 0.0 & 0.0 & 2829.0 & 0.0 & 0.0 & 22582.3 & 171.5 \\
Grand total & 8273.3 & 4166.5 & 741.0 & 9102.8 & 3899.5 & 593.5 & 4391.8 & 2816.8 & 395.0 & 3148.8 & 2209.8 & 511.5 & ---- & ---- \\
Grand mean & 827.3 & 416.7 & 74.1 & 910.3 & 390.0 & 59.4 & 439.2 & 281.7 & 39.5 & 314.9 & 221.0 & 51.2 & ---- & --- \\
Correlations & 0.80 & 0.47 & ---- & 0.61 & 0.35 & ---- & 0.47 & 0.06 & --- & 0.16 & 0.17 & ---- & ---- & ---- \\
\hline Tres wre
\end{tabular}

* Trees were harvested; $\mathrm{H}=$ H. cubana; $\mathrm{T}=$ T. tabaci; $\mathrm{N}=$ natural enemies; Shaded figures represent peak counts. 
Fluctuaciones poblacionales de $H$. cubana.

Primer ciclo (2012-2013):

Las densidades poblacionales de $H$. cubana (Cuadro 2 y Figura 2) se encontraron en niveles bajos de abundancia al inicio del ciclo en marzo (medias mensuales de 11.0, 7.3, 10.0 y 1.3 individuos / muestra de Cunningham, Nativa,

\section{Population fluctuation of $H$. cubana and T. tabaci:}

This part of the current investigation concentrate on the population dynamics of the most dangerous and abundant insect pests, H. cubana and T. tabaci under the effect of the natural enemies on different Leucaena genotypes (Cunningham, Native, K636, and KX2) were shown in Table 2 and 3 and illustrated graphically in Figure 2 and 3.

Cuadro 3. Número de medias mensuales de las plagas de leucaena $H$. cubana, T. tabaci y sus correlaciones con enemigos naturales, en los cuatro genotipos de Leucaena en el campo Xmatkuil durante (2013-2014).

Table 3. Monthly mean numbers of leucaena pests $\mathrm{H}$. cubana, T. tabaci and their correlations with natural enemies, on the four Leucaena genotypes at Xmatkuil farm during (2013-2014).

\begin{tabular}{|c|c|c|c|c|c|c|c|c|c|c|c|c|c|c|}
\hline \multirow{3}{*}{$\begin{array}{c}\text { Sampling } \\
\text { date } \\
\text { months }\end{array}$} & \multicolumn{12}{|c|}{ Mean numbers of individuals } & \multicolumn{2}{|c|}{ Grand mean } \\
\hline & \multicolumn{3}{|c|}{ Cunningham } & \multicolumn{3}{|c|}{ Nativa } & \multicolumn{3}{|c|}{ K636 } & \multicolumn{3}{|c|}{ KX2 } & \multirow[b]{2}{*}{$\mathrm{H}$} & \multirow[b]{2}{*}{$\mathrm{T}$} \\
\hline & $\mathrm{H}$ & $\mathrm{T}$ & $\mathrm{N}$ & $\mathrm{H}$ & $\mathrm{T}$ & $\mathrm{N}$ & $\mathrm{H}$ & $\mathrm{T}$ & $\mathrm{N}$ & $\mathrm{H}$ & $\mathrm{T}$ & $\mathrm{N}$ & & \\
\hline Jan., 2014 & $*$ & $*$ & $*$ & $*$ & * & * & $*$ & * & $*$ & $*$ & * & $*$ & $*$ & $*$ \\
\hline Feb. & $\begin{array}{c}8056.75 \\
*\end{array}$ & $\begin{array}{c}3.25 \\
*\end{array}$ & $\begin{array}{c}126.75 \\
*\end{array}$ & $\begin{array}{c}8880.75 \\
*\end{array}$ & 0 & $\begin{array}{c}82 \\
*\end{array}$ & & 0 & 131.25 & 580 & $\begin{array}{l}0 \\
*\end{array}$ & 152 & 22222.75 & 3.25 \\
\hline $\begin{array}{l}\text { Mar. } \\
\text { Apr. }\end{array}$ & 17.5 & 560.25 & 0 & 13.5 & 659.75 & 0 & 17.75 & 480.25 & 0 & 27 & 300.25 & $\begin{array}{l}* \\
0\end{array}$ & $\begin{array}{c}* \\
75.75\end{array}$ & 2000.5 \\
\hline May & $*$ & $*$ & $*$ & $*$ & $*$ & $*$ & $*$ & $*$ & $*$ & $*$ & $*$ & $*$ & $*$ & $*$ \\
\hline Jun. & 298.75 & 759.75 & 52.5 & 199 & 975.25 & 39.75 & 189.5 & 827 & 40.5 & 81 & 688.5 & 51.5 & 768.25 & 3250.5 \\
\hline Jul. & $*$ & $*$ & $*$ & $*$ & $*$ & $*$ & $*$ & $*$ & $*$ & $*$ & $*$ & $*$ & $*$ & $*$ \\
\hline Aug. & 174.5 & 477.5 & 131.5 & 136.25 & 415 & 63.75 & 139 & 213.5 & 46 & 42.75 & 81.5 & 63.75 & 492.5 & 1187.5 \\
\hline Sept. & $*$ & $*$ & * & $*$ & $*$ & $*$ & $*$ & $*$ & $*$ & $*$ & $*$ & $*$ & $*$ & * \\
\hline Oct., 2014 & 75.75 & 123.75 & 3 & 82.75 & 129.75 & 10.75 & 62 & 108.25 & 13.75 & 47.25 & 116.75 & 9.5 & 267.75 & 478.5 \\
\hline Grand total & 8623.25 & 1924.5 & 313.75 & 9312.25 & 2179.75 & 196.25 & 5113.5 & 1629 & 231.5 & 778 & 1187 & 276.75 & 5 & ---- \\
\hline Grand mean & 1724.65 & 384.9 & 62.75 & 1862.45 & 435.95 & 39.25 & 1022.7 & 325.8 & 46.3 & 155.6 & 237.4 & 55.35 & ---- & ---- \\
\hline Correlations & 0.572 & -0.22 & ---- & 0.70 & -0.35 & ---- & 0.94 & -0.45 & ---- & 0.91 & -0.36 & ---- & ---- & ---- \\
\hline
\end{tabular}

*Trees were harvested; $\mathrm{H}=H$. cubana; $\mathrm{T}=$ T. tabaci; $\mathrm{N}=$ natural enemies; Shaded figures represent peak counts.
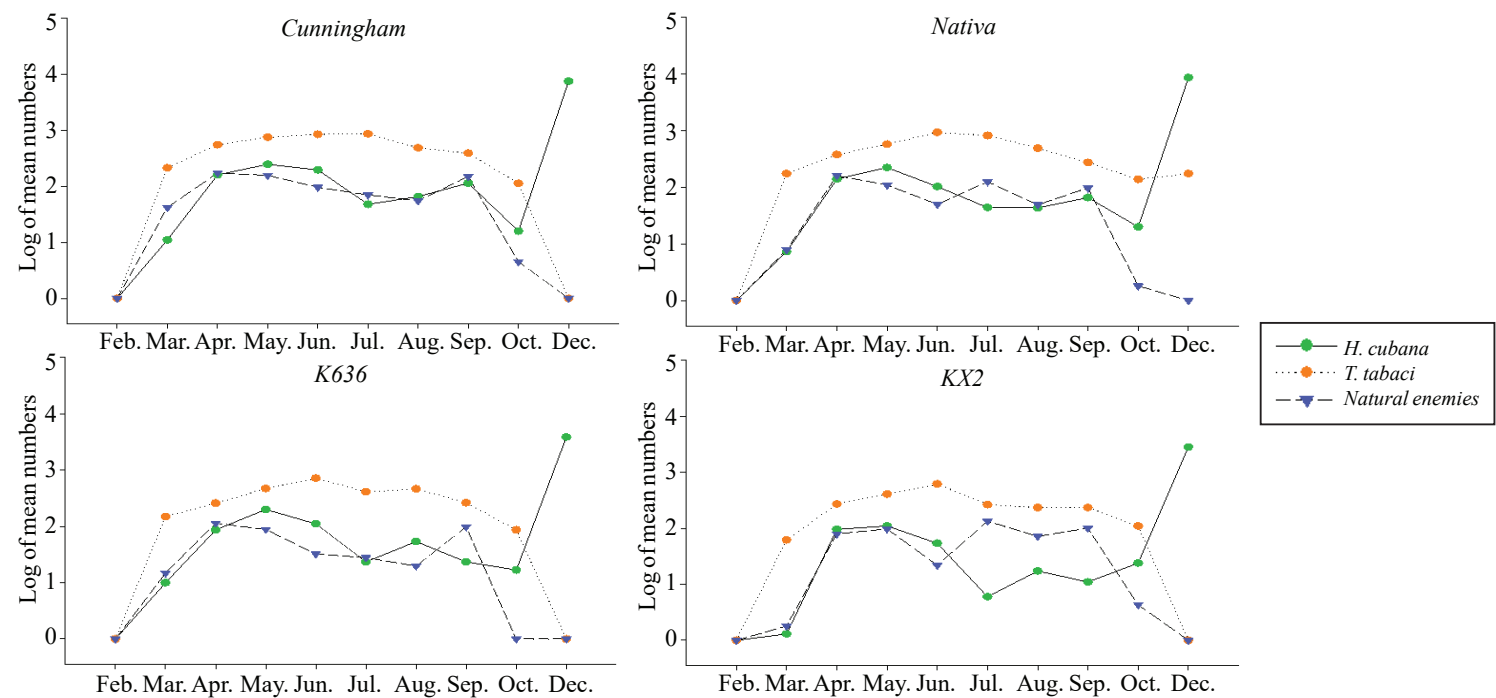

Figura 2. Dinámica poblacional de H. cubana, T. tabaci, y sus enemigos naturales en los genotipos de Leucaena durante 2012-2013 en el campo Xmatkuil, Yucatán, México.

Figure 2. Population dynamics of $H$. cubana, T. tabaci, and their natural enemies on Leucaena genotypes during 2012/2013 at Xmatkuil farm, Yucatan, Mexico. 

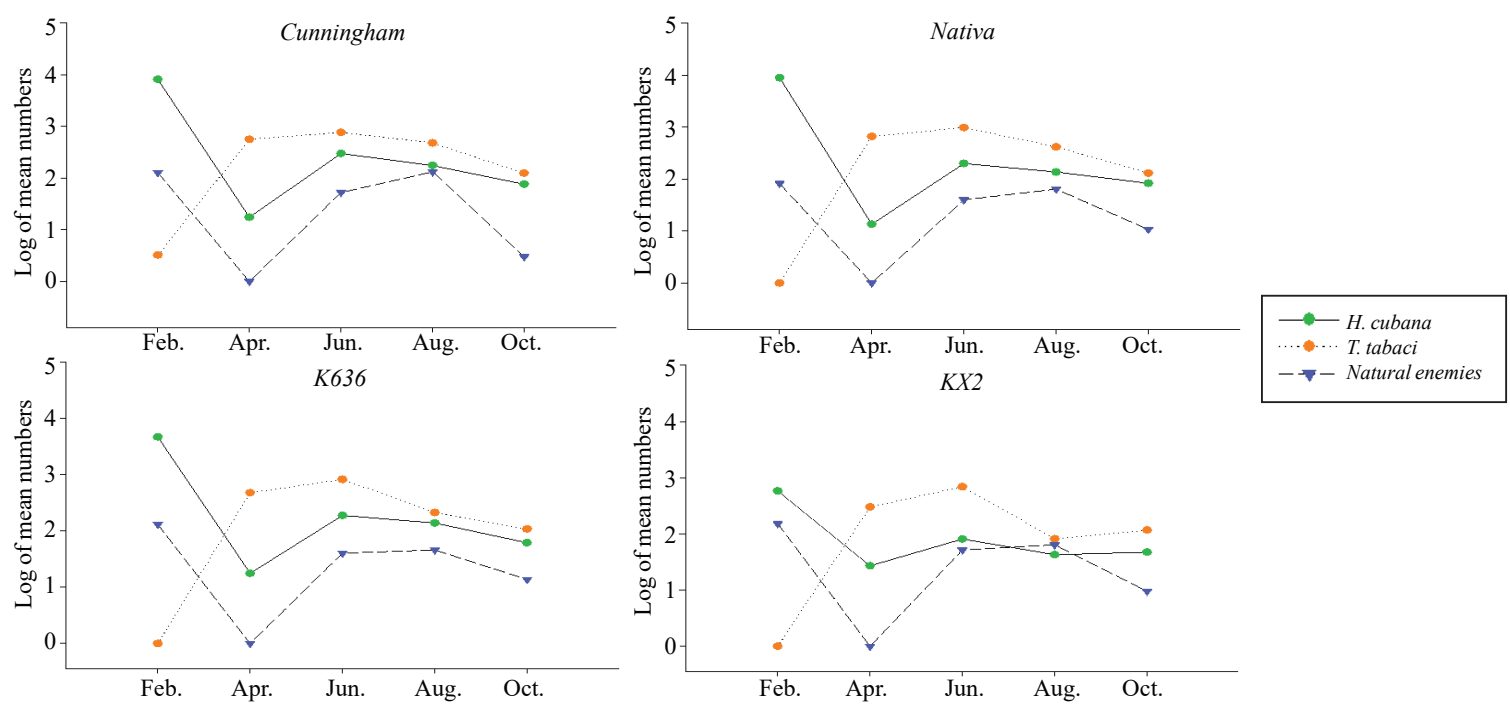

Figura 3. Dinámica poblacional de H. cubana, T. tabaci, y sus enemigos naturales en los genotipos de Leucaena durante 2013-2014 en el campo Xmatkuil, Yucatán, México.

Figure 3. Population dynamics of $H$. cubana, T. tabaci, and their natural enemies on Leucaena genotypes during 2013/2014 at Xmatkuil farm, Yucatan, Mexico.

K636, y KX2; respectivamente), luego aumento a niveles moderados de abundancia en abril (medias mensuales 159.0, 137.8, 87.0, 95.8 individuos / muestra). Después de esto, se produjeron dos picos antes de la primera cosecha en mayo (promedios mensuales 244.8, 194.8, 220.0 y 101.8) y junio (200.3, 111.0, 110.5 y 54.0 individuos / muestra) en Cunningham, Nativa, K636 y KX2, respectivamente como se muestra en (Cuadro 2; Figura 2). Posteriormente, el número de $H$. cubana disminuyo rápidamente en los siguientes cuatro meses (julio a octubre) hasta la cosecha en noviembre. Entonces, el tercer pico se registró en diciembre después de la cosecha de noviembre (medias mensuales 7422.3, 8465.0, 3866.0, 2829.0 individuos/ muestra) en Cunningham, Nativa, K636 y KX2, respectivamente.

\section{Segundo ciclo (2013-2014):}

Hubo una fuerte invasión de Psyllid (Cuadro 3 y Figura 3) al inicio de la temporada con diferencias significativas entre las densidades de Psyllid en los genotipos de Leucaena. El primer pico se produjo en febrero sobre los genotipos de Leucaena (medias mensuales 8056.75, 8880.75, 4705.25 y 580 individuos/muestra en Cunningham, Nativa, K636 y KX2; respectivamente). Después, las poblaciones de Psyllid se redujeron a los niveles más bajos de abundancia en abril con medias mensuales 17.5, 13.5, 17.75 y 27 en Cunningham, Nativa, K636 y KX2, respectivamente. E1 segundo pico se registró en junio (medias mensuales 298.75, 199, 189.5 y 81 individuos / muestra para Cunningham,

\section{Population fluctuations of $H$. cubana:}

\section{In the first season (2012-2013):}

The population densities of $H$. cubana (Table 2 and Figure 2 ) were in low levels of abundance at the beginning of the season during March (monthly means of 11.0, 7.3, 10.0 and 1.3 individuals/sample of Cunningham, Nativa, $\mathrm{K} 636$, and $\mathrm{KX} 2$; respectively), then increased to moderate levels of abundance during April (monthly means 159.0, 137.8, 87.0, 95.8 individuals/sample). After that, two peaks were occurred before the first harvest during May (monthly averages were 244.8, 194.8, 220.0, and 101.8) and June (200.3, 111.0, 110.5 and 54.0 individuals/sample) on Cunningham, Nativa, K636, and KX2; respectively as shown in (Table 2; Figure. 2). Afterwards, the numbers of H. cubana rapidly declined during the next four months (July and October) till harvest in November. Then, the third peak was recorded in December directly after the harvest of November (monthly means 7 422.3, 8 465.0, 3 866.0, 2 829.0 individuals-sample) on Cunningham, Nativa, K636 and $\mathrm{KX} 2$; respectively.

\section{In the second season (2013-2014):}

An intensive invasion of Psyllid population (Table 3 and Figure 3) occurred in the beginning of the season with obvious differences between Psyllid densities on Leucaena genotypes. The first peak occurred in February for Leucaena 
Nativa, K636 y KX2, respectivamente). Los niveles moderados de infestación se presentaron en agosto, después de que la población fluctuara lentamente en octubre sobre los genotipos de Leucaena (Cuadro 3 y la Figura 3).

\section{Fluctuación poblacional de T. tabaci:}

\section{Primer ciclo (2012-2013):}

T. tabaci mostró bajos niveles de infestación inicial (Cuadro 2 y Figura 2), en marzo 211.5, 171.5, 149.5 y 62.0 individuos/muestra para Cunningham, Nativa, K636 y KX2, respectivamente. Luego, la densidad poblacional empezó a aumentar lentamente en todos los genotipos de Leucaena, observando valores promedio altos de trips en Cunningham más que en otros genotipos en abril (medias mensuales $544.8,372.5,258.0$ y 272.5 individuos/muestra para Cunningham, Nativa, K636y KX2, respectivamente). Se registraron cuatro picos de trips en Cunningham, Nativay K636 durante mayo-agosto Las medias mensuales de mayo fueron 745.0, 567.3, 469.0; las medias de junio fueron 837.5, 918.3, 715.0; las medias de julio fueron 850.5, 809.0, 412.0; y las medias de agosto fueron 479.0,483.0,463.3 individuos/ muestra para Cunningham, Nativa y K636, respectivamente. También, se produjeron dos picos en KX2 en mayo y junio, con medias mensuales 409.0, 621.3 individuos/muestra, respectivamente. Después de esto hubo una disminución de la densidad poblacional a lo largo de los siguientes meses. La plaga desapareció por completo en diciembre después de la primera cosecha.

\section{Segundo ciclo (2013-2014):}

La población de trips se encontraban en niveles bajos de abundancia (Cuadro 3 y Figura 3) en febrero para los genotipos de Leucaena (medias mensuales 3.25, 0.0, 0.0 y 0.0 individuos/muestra en Cunningham, Nativa, K636 y KX2, respectivamente). Posteriormente, la población de trips presento dos picos en abril y junio. Las los valores medios en abril fueron $560.25,659.75,480.25$ y 300.25 para Cunningham, Nativa, K636 y KX2, respectivamente. Los valores medios del segundo pico en junio fueron $759.75,975.25,827$ y 688.5 individuos/muestra para los mismos genotipos respectivamente. A partir de entonces, se registraron niveles moderados de abundancia en agosto (medias mensuales 477.5, 415,213.5 y 81.5 en Cunningham, Nativa, K636 y KX2, respectivamente), después de que las poblaciones se redujeran al finales de octubre en casi todos los genotipos de Leucaena. genotypes (monthly means were $8056.75,8880.75,4705.25$ and 580 individuals-sample on Cunningham, Nativa, K636, and KX2; respectively).Afterwards, the populations of Psyllid were in lowest levels of abundance in April with monthly means 17.5, 13.5, 17.75 and 27 on Cunningham, Nativa, $\mathrm{K} 636$, and KX2; respectively. The second peak recorded in June (monthly means were 298.75, 199, 189.5 and 81 individuals/sample on Cunningham, Nativa, K636, and KX2; respectively). The moderate levels of infestations occurred in August, after that the population fluctuated slowly at October in Leucaena genotypes (Table 3 and Figure 3).

\section{Population fluctuations of T. tabaci:}

\section{In the first season (2012-2013):}

T. tabaci showed low levels of initial infestation (Table 2 and Figure 2) in March 211.5, 171.5, 149.5, and 62.0 individuals/sample on Cunningham, Nativa, K636 and KX2; respectively. Then, the population density grew up slowly on all Leucaena genotypes, with observation of high mean numbers of Thrips on Cuninngham more than other genotypes during April (monthly means were 544.8, 372.5; 258.0 and 272.5 individuals/sample on Cunningham, Nativa, K636 and KX2; respectively). Four peaks of Thrips were recorded on Cunningham, Nativa and K636 during May to Aug. The monthly means of May were 745.0, 567.3, 469.0; means of June were 837.5, 918.3, 715.0; means of July were 850.5, 809.0, 412.0; and means of August were 479.0, 483.0, 463.3 individuals/sample on Cunningham, Nativa and K636; respectively. Besides, two peaks were occurred on KX2 in May and June with monthly means 409.0, 621.3 individuals/ sample; respectively. After that a sharp declines of population densities occurred throughout the following months. Thepest totally disappeared in December after the $1^{\text {st }}$ harvest.

\section{In the second season (2013-2014):}

Thrips population were in low levels of abundance (Table 3 and Figure 3) in February for Leucaena genotypes (monthly means were 3.25, 0.0, 0.0 and 0.0 individuals/sample of Cunningham, Nativa, K636, and KX2; respectively). Afterwards, the population of Thrips showed two peaks in April and June. The mean numbers in April were 560.25, $659.75,480.25$ and 300.25 on Cunningham, Nativa, K636, and KX2; respectively. The means of the second peak of June were $759.75,975.25,827$ and 688.5 individuals/sample on the same respective genotypes. Thereafter, the moderate levels of abundance were recorded in August (monthly 


\section{El estudio de correlación entre los enemigos naturales y plagas:}

El coeficiente de correlación fue mayor entre los enemigos naturales y $H$. cubana que en $T$. tabaci para los genotipos de Leucaena durante el estudio.

\section{Durante el primer ciclo (2012-2013):}

El Psyllid Leucaena; H. cubana mostró una alta correlación con los enemigos naturales (Cuadro 2) para Cunningham $(\mathrm{r}=0.80)$, débil para KX2 $(\mathrm{r}=0.16)$ y moderada para Nativa y K636 (r=0.61 y 0.47 ) respectivamente. Por otro lado, la correlación fue alta entre $T$. tabaci y enemigos naturales de Cunningham $(\mathrm{r}=0.47)$, débil para K636( $\mathrm{r}=0.06)$, y moderada para Nativa y KX2 ( $\mathrm{r}=0.35$ y 0.17$)$ respectivamente.

\section{Durante el segundo ciclo (2013- 2014):}

Las correlaciones (Cuadro 3) entre enemigos naturales y Psyllid fueron altas ( $\mathrm{r}=0.94$ y 0.91 , para K636 y KX2, respectivamente), se registró una relación moderada para Nativa $(r=0.70)$, y débil para Cunningham $(r=0.572)$. Sin embargo, la correlación entre los enemigos naturales y trips fueron negativos en los genotipos de Leucaena.

\section{Discusión}

Las bajas fluctuaciones poblacionales de ambas plagas $H$. cubana y T. tabaci fueron similares al inicio de la temporada (Cuadro 1) con una clara diferencia entre su distribución espacial, debido al efecto de los enemigos naturales y los procesos de cosecha (antes y después de la cosecha).

La mayor incidencia de ambas plagas fue entre marzo y diciembre. Las densidades poblacionales de $T$. tabaci antes de la cosecha fueron superiores a $H$. cubana (Cuadro 2), ya que al inicio del experimento los árboles no eran lo bastante joven ( 2 años y 3 meses de edad) y nunca habían sido cosechados y T. tabaci no se ve afectado por la edad del hospedero, a diferencia de $H$. cubana que prefieren sólo hojas nuevas más que hojas viejas, lo que fomenta a altas densidades poblacionales de T. tabaci invadir hojas viejas de Leucaena más que H. cubana. Estos resultados concuerdan con Geigery Andrew (2000) y Chazeau et al. (1989) quienes reportaron que las poblaciones de $\mathrm{H}$. Cubana incrementaron sólo en la presencia de hojas jovenes y brotes nuevos de means 477.5, 415, 213.5 and 81.5 on Cunningham, Nativa, $\mathrm{K} 636$, and KX2; respectively), after that the populations were reduced at the end of the season in October on almost Leucaena genotypes.

\section{The correlation study between natural enemies and pests:}

The correlation coefficient was stronger between natural enemies and $H$. cubana than $T$. tabaci on Leucaena genotypes during the seasons of study.

\section{During The first season (2012-2013):}

The Leucaena Psyllid; H. cubana showed the strong correlation with natural enemies (Table 2) for Cunningham $(\mathrm{r}=0.80)$, weak for KX2 $(\mathrm{r}=0.16)$, and moderate $(\mathrm{r}=0.61$ and 0.47 ) for Nativa and K636; respectively. On the other side, the correlation was strong between T. tabaci and natural enemies for Cunningham $(\mathrm{r}=0.47)$, weak for K636 $(\mathrm{r}=0.06)$, and the moderate ( $\mathrm{r}=0.35$ and 0.17$)$ for Nativa and $\mathrm{KX} 2$; respectively.

\section{During the second season (2013-2014):}

The correlations (Table 3 ) between natural enemies and Psyllid were strong ( $r=0.94$ and 0.91 , for K636 and KX2 respectively), the moderate relation was recorded for Nativa $(\mathrm{r}=0.70)$, and weak for Cunningham ( $\mathrm{r}=0.572)$. However, the correlation between natural enemies and Thrips were negative in Leucaena genotypes.

\section{Discussion}

The slow population fluctuations of both pests $H$. cubana and T. tabaci were similar at the beginning of the season (Table 1) with a clear difference between their spatial distribution, due to the effect of natural enemy's agents and the harvest processes (before and after harvest).

The main prevalence of both pests fell between March and December. The population densities of $T$. tabaci before harvest were higher than H. cubana (Table 2), because in the beginning of the experiment the trees weren't young enough ( 2 years and 3 months old) and never harvested before and T. tabaci is not affected by the host age, in contrast with $H$. cubana which prefer only new leaves more than old leaves, 
Leucaena. Por lo que hubo un aumento evidente en las poblaciones del Psyllid durante las cosechas en la segunda temporada (Cuadro 3) continuando las mismas diferencias entre ambas poblaciones plaga.

\section{H. cubana:}

\section{Primer ciclo (2012-2013):}

Los individuos de H. cubana presentaron tres generaciones al año, dos antes de la cosecha en mayo y junio, y el tercer pico en diciembre después de la cosecha. Porque hubo una reducción evidente en las poblaciones de enemigos naturales durante los picos en comparación con los números reales del Psyllid. Esto se puede deber a la superposición de la nueva generación y los efectos de las altas temperaturas en la temporada de sequía durante mayo y junio en Mérida, Yucatán, las cuales reducen las poblaciones de enemigos naturales. Estos resultados concuerdan con lo señalado por Funasaki et al. (1989), quien afirma que C. coeruleus no puede establecer en muchas de sequía. Por lo tanto, los números de Psyllid aumentaron más que los enemigos naturales, y esto afecto a otros agentes de control biológico como $P$. yaseen que no pudo superar los picos de Psyllid.

Geiger y Andrew (2000) mencionaron que $P$. yaseen nunca tuvo éxito en la regulación de las poblaciones de Psyllid en presencia de altas poblaciones de Psyllid. En consecuencia, los picos se presentaron cuando la población Psyllid estaba fuera de control durante la ausencia de agentes de control biológico, ya que los agentes de enemigos naturales controlan automáticamente las poblaciones de Psyllid, de acuerdo con los resultados de (Bray 1994; Napompeth 1994; Geiger et al., 1995; Valenciaga et al., 1999; Singh, 2004; Shivankar et al., 2010). El tercer pico después de la cosecha podría haber ocurrió debido a la producción de hojas jóvenes de Leucaena, lo cual sustento alrededor de $98 \%$ de la infestación de H. cubana, porque hojas juveniles estimulan a las hembras para la puesta de huevos. Esto concuerda con lo reportado por (Chazeau et al., 1989).

\section{Segundo ciclo (2013-2014):}

La población Psyllid baja durante genotipos no cosechada de Leucaena en febrero de 2013 de la primera temporada, y el brote de plagas en todos los genotipos de Leucaena durante febrero de 2014 hizo hincapié en que la cosecha fue el motivo de la primera cumbre, porque los nuevos y juveniles hojas de genotipos de Leucaena después de la cosecha the thing which encourage high population densities of $T$. tabaci to invade old Leucaena more than $H$. cubana. These results are in agreement with Geiger and Andrew (2000) and Chazeau et al. (1989) who reported that, H. cubana populations boomed only in the presence of young Leucaena leaves and new shoots. Therefore, an obvious increase of psyllid populations occurred during the successive harvests in the second season (Table 3) with the continued existence of the same differences between both pests populations.

\section{H. cubana:}

\section{In the first season (2012-2013):}

H. cubana individuals showed three generations a year, two before harvest in May and June, and the third peak in December after harvest. Because, an obvious reductions were observed in natural enemies populations during peaks times compared by the actual numbers of Psyllid. This could be due to the new generation overlapping and the effects of high temperatures in the dry seasons during May and June at Merida, Yucatan which reduce the populations of natural enemies. These results are in agreement with those reported by Funasaki et al. (1989) who stated that $C$. coeruleus failed to be established in many seasonal dry areas. Therefore, Psyllid numbers increased more than natural enemy's agents, and this affected other biological control agents such as P.yaseeni which can't overcomePsyllid peaks during high Psyllid numbers.

Geiger and Andrew (2000) stated that $P$. yaseeni never succeeded in regulating Psyllid populations in the presence of high Psyllid populations. Accordingly, the peaks were occurred when the Psyllid population was out of control during the absence of biological control agents, because natural enemy's agents automatically control Psyllids populations, according to the findings of (Bray 1994; Napompeth 1994; Geiger et al. 1995; Valenciaga et al. 1999; Singh 2004; Shivankar et al. 2010). The third peak after harvest could be occurred due to the young leaves production of Leucaena, which supported about $98 \%$ of H. cubana infestation, because juvenile leaves encourage females for egg-laying. This is in accordance with those reported by (Chazeau et al., 1989).

\section{In the second season (2013-2014):}

The low Psyllid population during non-harvested Leucaena genotypes at February, 2013of the first season, and the outbreak of pest in all Leucaena genotypes during February, 
apoyado hembras Psyllid para la puesta de huevos. Esto está de acuerdo con (Chazeau et al., 1989). Por lo tanto, los enemigos naturales no fueron capaces de controlar el brote de Psyllid porque hubo diferencias claras entre las plagas y las densidades de enemigos naturales en todos los genotipos de Leucaena (Cuadro 3). Sin embargo, esta diferencia impide la eficacia de muchos enemigos naturales. De acuerdo con (Geiger y Andrew 2000).

El segundo pico en junio, que se presentó en el ciclo anterior en los árboles no cosechados, ocurrió debido al tiempo normal de superposición de la generación. En consecuencia, la población se redujo en agosto y octubre, debido a las bajas temperaturas de estos meses en Mérida, Yucatán, México. Los mismos resultados fueron reportados por Anónimo (2014), donde la reproducción y el desarrollo del Psyllid se detuvo o disminuyo por la temporada de frío y la mayoría de las especies tienen de 3 a 5 generaciones al año y algunas especies pueden tener una generación al año.

\section{T. tabaci:}

\section{Primer ciclo (2012-2013):}

Los cuatro picos de T. tabaci en Cunningham, Nativa y K636 de mayo a agosto y los dos picos en KX2 en mayo y junio, se puede deber a la cantidad de superposición de generaciones que dependen de factores climáticos (especialmente temperaturas máximas), planta hospedera, y el número de enemigos naturales. (Bastos et al., 1981) registraron cuatro picos de población de trips en Brasil, que representan cuatro generaciones de la plaga. Sin embargo, la variación en los resultados presentes puede atribuirse a las diferencias entre genotipos de Leucaena. Los valores totales de T. tabaci (Cuadro 2) se presentaron en los genotipos susceptibles Cunningham y Nativa. Estos resultados concuerdan con Castillo et al. (1997), quienes encontraron que las líneas más susceptibles fueron Cunningham (L. leucocephala) y Nativa (L. leucocephala x L. pallida) que mostraron resistencia moderada entre las diferentes accesiones de Leucaena ante plagas.

Además, las diferencias entre las fluctuaciones de los enemigos naturales de los genotipos de Leucaena, se puede explicar debido a la variación en el número de picos entre los genotipos de Leucaena.Además, parece que las poblaciones de T. tabaci estaban fuera de control bajo el efecto de los enemigos naturales, debido a la baja densidad de población de estos encontradas en Cunningham, Nativa y K636 en
2014 emphasized that the harvest was the reason for the first peak, because the new and juvenile leaves of leucaena genotypes after harvest supported Psyllids females for egglaying. This is in agreement with (Chazeau et al., 1989). Therefore, the natural enemies weren't able to control the outbreak of Psyllid because there were clear differences between pest and natural enemies' densities in all Leucaena genotypes (Table 3). However, this difference impedes the efficiency of many natural enemies. This is in accordance with (Geiger and Andrew 2000). The second peak of June which happened in the previous season under non-harvested trees occurred due to the normal time of overlapping generation. Consequently, the population reduced in August and October, due to the low temperatures during these months in Merida, Yucatán, Mexico. The same results were found by Anonymous (2014) where the reproduction and development of Psyllid were stopped or declined during cool weather and most species have 3 to 5 generations a year and some species may have one generation a year.

\section{T. tabaci:}

\section{In the first season (2012-2013):}

The four peaks of T. tabaci on Cunningham, Nativa, and K636 during May to Aug. and the two peaks of KX2 during May and Jun could be due to the numbers of overlapping generations which depend on the climatic factors (especially maximum temperature), host plant, and the numbers of natural enemies. (Bastos et al. 1981) recorded four population peaks of Thrips in Brazil which represented four generations of the pest. Nevertheless, the variation in the present results could be attributed to the differences between Leucaena genotypes. The highest grand total numbers of T. tabaci (Table 2) was occurred on the susceptible genotypes; Cunningham and Nativa. These are in agreement with Castillo et al.(1997), who found that, the most susceptible lines were Cunningham $(L$. leucocephala) and Nativa (L. leucocephalax L.pallida) which showed moderately resistance among different Leucaena accessions to pest infestation.

Besides, the differences between the fluctuations of the natural enemies on Leucaena genotypes, this may explain such variation in the numbers of peaks among Leucaena genotypes. Additionally, it seems that T. tabaci populations were out of control under the effect of natural enemies, because low population densities of the natural enemies were found on Cunningham, Nativa, and K636 compared with the numbers of $T$. tabaci during the four peaks, and as 
comparación con los números de T. tabaci durante los cuatro picos, y como sabe que los enemigos naturales regulan automáticamente el número de plagas de Leucaena (Bray 1994; Napompeth 1994; Singh 2004; Shivankar et al., 2010).

\section{Segundo ciclo (2013-2014):}

Los trips fluctuaron lentamente (Cuadro 3) al inicio del ciclo en febrero para los genotipos de Leucaena, debido a que las altas densidades de enemigos naturales mantuvieron las poblaciones de trips bajo control. Posteriormente, la población de la plaga aumentó debido a la total ausencia de enemigos naturales los cuales fomentaron la formación de picos en los genotipos de Leucaena en abril. Del mismo modo, en el mes junio, la baja población de enemigos naturales permitió el brote de plagas. Estos resultados concuerdan con Alston (2008) quien informó que la abundancia de enemigos naturales es suficiente para suprimir la población de trips.

\section{Conclusión}

Los arboles de Leucaena resultado de cosechas consecutivas cada dos meses fueronmejores que los árboles de leucaena que crecen libremente, ya que mostró bajos picos poblacionales de Psyllid y trips. Sin embargo, se recomiendan plantar los genotipos KX2 y K636 entre los genotipos de Leucaena estudiadas por su resistencia a las plagas, debido a la baja dinámica poblacional de ambas plagas. Los enemigos naturales lograron resultados satisfactorios en el control biológico de plagas, especialmente larvas y adultos del orden: Coleoptera. Por lo tanto, el cuidado de campos de Leucaena con enemigos naturales es laúnica manera para el control de plagas y así evitar el uso de insecticidas en árboles forrajeros para animales.

\section{References}

Alston, D. G. 2008. Onion Thrips (Thrips tabaci). Available at: http:// utahpests.usu.edu/IPM/htm/vegetables/vegetable-insectdisease/onion-thrips. (Accessed: September 2014).

Anonymous. 2014. Pests in Gardens and landscape, University of California Agriculture \& Natural Resources. Available at: http://www.ipm.ucdavis.edu/PMG/PESTNOTES/pn7423. html (Accessed: September 2014). it known that natural enemies automatically regulating the numbers of Leucaena pests (Shivankar et al. 2010; Singh 2004; Bray 1994; Napompeth 1994).

\section{In the second season (2013-2014):}

The Thrips fluctuated slowly (Table 3 ) in the beginning of the season in February for Leucaena genotypes, because the high densities of natural enemies kept Thrips populations under control.Afterwards, the population of the pest increased due to the total absence of natural enemies which encouraged peaks formation on Leucaena genotypes during April. Likewise, in the next month of June the low population of natural enmies permits pest outbreak. These results are in agreement with Alston (2008) who reported that the plenty of natural enemies are adequate to suppress Thrips population.

\section{Conclusion}

Leucaena trees resulted from consequence harvests every two months were better than free growing leucaena trees, because it showed low Psyllid and Thrips population peaks. However, KX2 and K636 genotypes are recommended for plantation among the studied Leucaena genotypes for their pest's resistance with an obvious low population's dynamics of both pests. Natural enemies achieved satisfactory results in biological control field for pests, specially larvae and adults of Order: Coleoptera. Therefore, caring with natural enemies in Leucaena fields is the only important way for pest control and avoiding insecticides utilization with such animal fodder tree.

\section{Acknowledgement}

I would like to express my deepest thanks to Consejo Nacional de Ciencia y Tecnología (CONACYT/ Mexico) which gave me the opportunity for obtaining a scholarship to conduct my Ph.D. researchers at Faculty of Veterinary Medicine and Animal Science, University of Yucatan (UADY), Merida, Yucatan, Mexico.

End of the English version 
Bastos, J.A.M.;Alves, V.P.O.; Magalhaes, B.J.A. and Oliveira,A.V.P. 1981. Survey of the population of the red grapevine Thrips Retithrips syriacus (Mayet, 1890) on grapevine. Fitossanidade. 5:1-6.

Bray, R. A. 1994. The leucaena psyllid. In: Forage Tree legumes in Tropical Agriculture Gutteridge, R.C. and H.M. Shelton (eds) (Department of Agriculture, The University of Queensland, Australia). CAB International, Oxford. 283-291 pp.

Castillo, A. C.; Cuyugan, O. C.; Forgary, S. and Shelton, H. M. 1997. Growth, psyllid resistance and forage quality of Leucaena leucocephala, L. pallid, L. diversifolia and F1 hybrid of $L$. leucocephala $x$ L. pallid. Tropical Grassland. 31:188-200.

Chazeau, J.; Bouye, E. and Bonnet de Larborgne, L.. 1989. Lutte biologique contre le psylle Heteropsylla cubana ravageur du faux mimosa Leucaena leucocephala en Nouvelle Calédonie. Orstom, Nouméa.

Food and Agriculture Organization of the United Nations (FAO). 2007. Forest Health \& Biosecurity Working Papers, OVERVIEW OF FOREST PESTS MALAWI. Working Paper FBS/22E, FAO, Rome, Italy. 8 p.

Funasaki, G. Y.; Lai,P.Y. and Nakahara,L.M. 1989. Status of natural enemies of Heteropsylla cubana Crawford (Homoptera: Psyllidae) in Hawaii. In: Leucaena Psyllid: Problems and Management. Napompeth, B. and K.G. MacDicken (eds). Proceedings Bogor, Indonesia Winrock International, Bangkok, Thailand. 153-158pp.

Funasaki, G.Y.; Lai, P.Y., and Nakahara, L.M. 1990. Status of natural enemies of Heteropsylla cubana Crawford (Homoptera: Psyllidae) in Hawaii, in Leucaena psyllid: problems and management. Proceedings of an International Workshop held in Bogor, Indonesia, January 1 6-21, 1989. F/FRED, IDRC, NFTA, Bangkok, Thailand, 153-158 pp.

Geiger, C. A.; Napompeth, B. and Van Den Beldt, R. J. 1995. An update on the status of the leucaena Psyllid in Southeast Asia. In: Leucaena: Opportunities and Limitations. Shelton, H.M., C.M. Piggin and J.L. Brewbaker. Canberra(eds). Australian Centre for Agricultural Research. 8-125 pp.

Geiger, C. A. and Andrew, G. P. 2000. Ecology of Heteropsylla cubana (Homoptera: Psyllidae): Psyllid Damage, Tree Phenology, Thermal Relations, and Parasitism in the Field. Environ. Entomol. 29:76-86.

Ghabn, A. A.A. E. 1948. Contribution to the knowledge of the biology of Thrips tabaci Lindeman in Egypt. Bulletin de la Societe Fouad Ier d'Entomologie. 32:123-174.
Heydon, D. and Affonso, M. 1990. Economic review of psyllid damage on leucaena in Southeast Asia and Australia. In: A report prepared for the Australian International Development Assistance Bureau (AIDAB), CAB International Development Services, Wallingford, Oxford, U.K. 129 p.

Napompeth, B. 1994. Leucaena psyllid in the Asia-Pacific region: implications for its management in Africa. In: Workshop Proceedings. LeucaenaPsyllid: a Threat to Agroforestry in Africa. FAO Corporate Document Repository Food and Agriculture Organization of the United Nations, Rome, Italy. $1-15 \mathrm{pp}$.

Nair, K. S. S. 2007. Tropical Forest Insect Pests. Ecology, Impact, and Management. Book. by Dr. Nair, K. S. S. ISBN: 9780521873321. [Accessed 3 Jan., 2014]. Available from URL: http:/ www.cambridge.org/9780521873321.

Olckers, T. 2011. Biological Control of Leucaena leucocephala (Lam.) de Wit (Fabaceae) in South Africa: A Tale of Opportunism, Seed Feeders and Unanswered Questions. African Entomology. 19(2):356-365.

Rao, M. R.; Singh, M.P. and Day, R. 2000. Insect pest problems in tropical agroforestry systems: Contributory factors and opportunities for management. Kluwer Academic Publisher, printed in Netherlands. Agroforest Systems. 50:243-277.

Sakimura, K. 1932. Life-history of Thrips tabaci Lindeman, on Emilia sagittata and its host plant range in Hawaii. Journal of Economic Entomology. 25:884-891.

Shivankar, V. J. and Rao, C. N. 2010. Psyllid and pest management. Pest Management in Horticultural Ecosystems. 16:1-4.

Singh, S. P. 2004. Some Successes Stories in Classical Biological Control of Agriculture Pests in India. Asia-Pacific Association of Agricultural Research Institutions (APAARI) FAO-RAP, Maliwan Mansion 39 Phra Atit Road Bangkok 10200, Thailand. [Accessed 7 Feb., 2014]. Available from URL: http:// http://www.apaari.org/wp-content/uploads/2009/05/ ss_2004_02.pdf.

USAID. 1991. Environment Assessment of the classical biological control of the leucaena psyllid in Indonesia, Laos, Malaysia, Nepal, Philippines and Thailand. USAID Report, Washington DC. plus appendices. $94 \mathrm{p}$.

Valenciaga, N.; Barrientos, A. and Mora, C. 1999. Performance of the beneficial entomofauna in Leucaena leucocephala (Lam.) de Wit areas. Cuban Journal of Agricultural Science. 33:319-24. 